PROCEEDINGS OF THE

AMERICAN MATHEMATICAL SOCIETY

Volume 135, Number 6, June 2007, Pages 1597-1606

S 0002-9939(06)08842-3

Article electronically published on December 27, 2006

\title{
ON THE REGULARITY OF PRODUCTS AND INTERSECTIONS OF COMPLETE INTERSECTIONS
}

\author{
MARC CHARDIN, NGUYEN CONG MINH, AND NGO VIET TRUNG \\ (Communicated by Michael Stillman)
}

Abstract. This paper proves the formulae

$$
\begin{aligned}
\operatorname{reg}(I J) & \leq \operatorname{reg}(I)+\operatorname{reg}(J), \\
\operatorname{reg}(I \cap J) & \leq \operatorname{reg}(I)+\operatorname{reg}(J)
\end{aligned}
$$

for arbitrary monomial complete intersections $I$ and $J$, and provides examples showing that these inequalities do not hold for general complete intersections.

\section{INTRODUCTION}

Let $S$ be a polynomial ring over a field $k$. For a finitely generated graded $S$ module $M$ let

$$
a_{i}(M):=\max \left\{\mu \mid H_{\mathfrak{m}}^{i}(M)_{\mu} \neq 0\right\}
$$

if $H_{\mathfrak{m}}^{i}(M) \neq 0$ and $a_{i}(M):=-\infty$ otherwise, where $H_{\mathfrak{m}}^{i}(M)$ denotes the $i$ th local cohomology module of $M$ with respect to the graded maximal ideal $\mathfrak{m}$ of $S$. Then the Castelnuovo-Mumford regularity (or regularity for short) of $M$ is defined as the invariant

$$
\operatorname{reg}(M):=\max _{i}\left\{a_{i}(M)+i\right\} .
$$

It is of great interest to have good bounds for the regularity $\mathrm{BaM}$.

The regularity of products of ideals was first studied by Conca and Herzog $[\mathrm{CoH}]$. They found some special classes of ideals $I$ and $J$ for which the following formula holds:

$$
\operatorname{reg}(I J) \leq \operatorname{reg}(I)+\operatorname{reg}(J)
$$

(see also [Si]). In particular, they showed that $\operatorname{reg}\left(I_{1} \cdots I_{d}\right)=d$ for any set of ideals $I_{1}, \ldots, I_{d}$ generated by linear forms. These results led them to raise the question whether the formula

$$
\operatorname{reg}\left(I_{1} \cdots I_{d}\right) \leq \operatorname{reg}\left(I_{1}\right)+\cdots+\operatorname{reg}\left(I_{d}\right)
$$

holds for any set of complete intersections $I_{1}, \ldots, I_{d}[\mathrm{CoH}$, Question 3.6]. Note that this formula does not hold for arbitrary monomial ideals. For instance, Terai and Sturmfels (see [St]) gave examples of monomial ideals $I$ such that $\operatorname{reg}\left(I^{2}\right)>2 \operatorname{reg}(I)$.

On the other hand, Sturmfels conjectured that $\operatorname{reg}\left(I_{1} \cap \cdots \cap I_{d}\right) \leq d$ for any set of ideals $I_{1}, \ldots, I_{d}$ generated by linear forms. This conjecture was settled in the

Received by the editors March 7, 2005 and, in revised form, February 6, 2006.

2000 Mathematics Subject Classification. Primary 13D02.

The second author was partially supported by the National Basic Research Program of Vietnam. 
affirmative by Derksen and Sidman [DS. Their proof was inspired by the work of Conca and Herzog. So one might be tempted to ask whether the formula

$$
\operatorname{reg}\left(I_{1} \cap \cdots \cap I_{d}\right) \leq \operatorname{reg}\left(I_{1}\right)+\cdots+\operatorname{reg}\left(I_{d}\right)
$$

holds for any set of complete intersections $I_{1}, \ldots, I_{d}$.

The following result shows that these questions have positive answers in the monomial case and we shall see that there are counter-examples in the general case.

Theorem 1.1. Let $I$ and $J$ be two arbitrary monomial complete intersections. Then

$$
\begin{aligned}
\operatorname{reg}(I J) & \leq \operatorname{reg}(I)+\operatorname{reg}(J), \\
\operatorname{reg}(I \cap J) & \leq \operatorname{reg}(I)+\operatorname{reg}(J) .
\end{aligned}
$$

Both formulae follow from a more general bound for the regularity of a larger class of ideals constructed from $I$ and $J$ (Theorem 3.1). The proof is a bit intricate. It is based on a bound for the regularity of a monomial ideal in terms of the degree of the least common multiple of the monomial generators and the height of the given ideal.

We are not able to extend the first formula to more than two monomial complete intersections. But we find another proof which extends the second formula to any finite set of monomial complete intersections (Theorem 3.3). We would like to mention that the first formula was already proved in the case where one of the ideals $I, J$ is generated by two elements by combinatorial methods in $\mathrm{M}$.

In the last section, we give a geometric approach for constructing examples of complete intersection ideals for which the inequalities $\operatorname{reg}(I J) \leq \operatorname{reg}(I)+\operatorname{reg}(J)$ and/or $\operatorname{reg}(I \cap J) \leq \operatorname{reg}(I)+\operatorname{reg}(J)$ fail. We show for instance the following.

Theorem 1.2. Let $Y$ in $\mathbb{P}^{3}$ be a curve which is defined by at most 4 equations at the generic points of its irreducible components. Consider 4 elements $f_{1}, f_{2}, f_{3}, f_{4}$ in $I_{Y}$ such that $I:=\left(f_{1}, f_{2}\right)$ and $J:=\left(f_{3}, f_{4}\right)$ are complete intersection ideals and $I_{Y}$ is the unmixed part of $I+J$. Assume that $\varepsilon:=\min \left\{\mu \mid H^{0}\left(Y, \mathcal{O}_{Y}(\mu)\right) \neq 0\right\}<0$. Then

$$
\operatorname{reg}(I J)=\operatorname{reg}(I)+\operatorname{reg}(J)-\varepsilon-1 .
$$

A similar construction is explained for $I \cap J$. As a consequence, many families of curves with sections in negative degrees give rise to counter-examples for the considered inequalities. In the examples we give, $I$ is a monomial ideal and $J$ is either generated by one binomial or by one monomial and one binomial.

\section{Preliminaries}

Let us first introduce some conventions. For any monomial ideal we can always find a minimal basis consisting of monomials. These monomials will be called the monomial generators of the given ideal. Moreover, for a finite set of monomials $A_{i}=x_{1}^{a_{i 1}} \cdots x_{n}^{a_{i n}}$, we call the monomial $x_{1}^{\max _{i}\left\{a_{i 1}\right\}} \cdots x_{n}^{\max _{i}\left\{a_{i n}\right\}}$ the least common multiple of the monomials $A_{i}$.

The key point of our approach is the following bound for the regularity of arbitrary monomial ideals. 
Lemma 2.1 ([T, Lemma 3.1]). Let I be a monomial ideal. Let $F$ denote the least common multiple of the monomial generators of $I$. Then

$$
\operatorname{reg}(I) \leq \operatorname{deg} F-\operatorname{ht}(I)+1 .
$$

This bound is an improvement of the bound $\operatorname{reg}(I) \leq \operatorname{deg} F-1$ given by Bruns and Herzog in $[\mathrm{BrH}$, Theorem 3.1(a)].

If we apply Lemma 2.1 to the product and the intersection of monomial ideals, we get

$$
\begin{gathered}
\operatorname{reg}\left(I_{1} \cdots I_{d}\right) \leq \sum_{j=1}^{d} \operatorname{deg} F_{j}-\operatorname{ht}\left(I_{1} \cdots I_{d}\right)+1, \\
\operatorname{reg}\left(I_{1} \cap \cdots \cap I_{d}\right) \leq \sum_{j=1}^{d} \operatorname{deg} F_{j}-\operatorname{ht}\left(I_{1} \cap \cdots \cap I_{d}\right)+1,
\end{gathered}
$$

where $F_{j}$ denotes the least common multiple of the monomial generators of $I_{j}$. If $I_{1}, \ldots, I_{d}$ are complete intersections, then $\operatorname{reg}\left(I_{j}\right)=\operatorname{deg} F_{j}-\operatorname{ht}\left(I_{j}\right)+1$, whence

$$
\begin{gathered}
\operatorname{reg}\left(I_{1} \cdots I_{d}\right) \leq \sum_{j=1}^{d} \operatorname{reg}\left(I_{j}\right)+\sum_{j=1}^{d} \operatorname{ht}\left(I_{j}\right)-\operatorname{ht}\left(I_{1} \cdots I_{d}\right)-d+1, \\
\operatorname{reg}\left(I_{1} \cap \cdots \cap I_{d}\right) \leq \sum_{j=1}^{d} \operatorname{reg}\left(I_{j}\right)+\sum_{j=1}^{d} \operatorname{ht}\left(I_{j}\right)-\operatorname{ht}\left(I_{1} \cap \cdots \cap I_{d}\right)-d+1 .
\end{gathered}
$$

These bounds are worse than the bounds in the aforementioned questions. However, the difference is not so great.

To get rid of the difference in the case $d=2$ we need the following consequence of Lemma 2.1.

Corollary 2.2. Let $I$ be a monomial complete intersection and $Q$ an arbitrary monomial ideal (not necessarily a proper ideal of the polynomial ring $S$ ). Then

$$
\operatorname{reg}(I: Q) \leq \operatorname{reg}(I) \text {. }
$$

Proof. Let $F$ denote the product of the monomial generators of $I$. Since every monomial generator of $I: Q$ divides a monomial generator of $I$, the least common multiple of the monomial generators of $I: Q$ divides $F$. Applying Lemma 2.1 we get

$$
\operatorname{reg}(I: Q) \leq \operatorname{deg} F-\operatorname{ht}(I: Q)+1 \leq \operatorname{deg} F-\operatorname{ht} I+1=\operatorname{reg}(I) .
$$

We will decompose the product and the intersection of two monomial ideals as a sum of smaller ideals and apply the following lemma to estimate the regularity.

Lemma 2.3. Let $I$ and $J$ be two arbitrary homogeneous ideals. Then

$$
\begin{aligned}
\operatorname{reg}(I+J) & \leq \max \{\operatorname{reg}(I), \operatorname{reg}(J), \operatorname{reg}(I \cap J)-1\}, \\
\operatorname{reg}(I \cap J) & \leq \max \{\operatorname{reg}(I), \operatorname{reg}(J), \operatorname{reg}(I+J)+1\} .
\end{aligned}
$$

Moreover, $\operatorname{reg}(I \cap J)=\operatorname{reg}(I+J)+1$ if $\operatorname{reg}(I+J)>\max \{\operatorname{reg}(I), \operatorname{reg}(J)\}$ or if $\operatorname{reg}(I \cap J)>\max \{\operatorname{reg}(I), \operatorname{reg}(J)\}+1$. 
Proof. The statements follow from the exact sequence

$$
0 \longrightarrow I \cap J \longrightarrow I \oplus J \longrightarrow I+J \longrightarrow 0
$$

and the well-known relationship between regularities of modules of an exact sequence (see e.g. [E, Corollary 20.19]).

\section{MAin REsults}

We will prove the following general result.

Theorem 3.1. Let $I$ and $J$ be two arbitrary monomial complete intersections. Let $f_{1}, \ldots, f_{r}$ be the monomial generators of $I$. Let $Q_{1}, \ldots, Q_{r}$ be arbitrary monomial ideals. Then

$$
\operatorname{reg}\left(f_{1}\left(J: Q_{1}\right)+\cdots+f_{r}\left(J: Q_{r}\right)\right) \leq \operatorname{reg}(I)+\operatorname{reg}(J) .
$$

The formulae of Theorem 1.1 follow from the above result because

$$
\begin{aligned}
I J & =f_{1} J+\cdots+f_{r} J=f_{1}(J: S)+\cdots+f_{r}(J: S), \\
I \cap J & =\left(f_{1}\right) \cap J+\cdots+\left(f_{r}\right) \cap J=f_{1}\left(J: f_{1}\right)+\cdots+f_{r}\left(J: f_{r}\right) .
\end{aligned}
$$

Proof. If $r=1$, we have to prove that

$$
\operatorname{reg}\left(f_{1}\left(J: Q_{1}\right)\right) \leq \operatorname{deg} f_{1}+\operatorname{reg}(J) .
$$

It is obvious that

$$
\operatorname{reg}\left(f_{1}\left(J: Q_{1}\right)\right) \leq \operatorname{deg} f_{1}+\operatorname{reg}\left(J: Q_{1}\right) .
$$

By Corollary 2.2 we have $\operatorname{reg}\left(J: Q_{1}\right) \leq \operatorname{reg}(J)$, which implies the assertion.

If $r>1$, using induction we may assume that

$$
\begin{aligned}
\operatorname{reg}\left(\sum_{i=1}^{r-1} f_{i}\left(J: Q_{i}\right)\right) & \leq \operatorname{reg}\left(f_{1}, \ldots, f_{r-1}\right)+\operatorname{reg}(J), \\
\quad \operatorname{reg}\left(f_{r}\left(J: Q_{r}\right)\right) & \leq \operatorname{deg} f_{r}+\operatorname{reg}(J) .
\end{aligned}
$$

Since $f_{1}\left(J: Q_{1}\right), \ldots, f_{r-1}\left(J: Q_{r-1}\right)$ are monomial ideals, we have

$$
\left(\sum_{i=1}^{r-1} f_{i}\left(J: Q_{i}\right)\right): f_{r}=\sum_{i=1}^{r-1}\left(f_{i}\left(J: Q_{i}\right): f_{r}\right) .
$$

Since $f_{1}, \ldots, f_{r}$ is a regular sequence, $f_{i}\left(J: Q_{i}\right): f_{r}=f_{i}\left(J: f_{r} Q_{i}\right)$. Therefore,

$$
\begin{aligned}
\left(\sum_{i=1}^{r-1} f_{i}\left(J: Q_{i}\right)\right) \cap f_{r}\left(J: Q_{r}\right) & =f_{r}\left[\left(\sum_{i=1}^{r-1} f_{i}\left(J: Q_{i}\right): f_{r}\right) \cap\left(J: Q_{r}\right)\right] \\
& =f_{r}\left[\left(\sum_{i=1}^{r-1} f_{i}\left(J: f_{r} Q_{i}\right)\right) \cap\left(J: Q_{r}\right)\right] \\
& =f_{r}\left[\sum_{i=1}^{r-1} f_{i}\left(\left(J: f_{r} Q_{i}\right) \cap\left(J: f_{i} Q_{r}\right)\right)\right] \\
& =f_{r}\left[\sum_{i=1}^{r-1} f_{i}\left(J:\left(f_{r} Q_{i}+f_{i} Q_{r}\right)\right)\right] .
\end{aligned}
$$


From this it follows that

$$
\operatorname{reg}\left(\left(\sum_{i=1}^{r-1} f_{i}\left(J: Q_{i}\right)\right) \cap f_{r}\left(J: Q_{r}\right)\right) \leq \operatorname{deg} f_{r}+\operatorname{reg}\left(\sum_{i=1}^{r-1} f_{i}\left(J:\left(f_{r} Q_{i}+f_{i} Q_{r}\right)\right)\right) .
$$

Using induction we may assume that

$$
\operatorname{reg}\left(\sum_{i=1}^{r-1} f_{i}\left(J:\left(f_{r} Q_{i}+f_{i} Q_{r}\right)\right)\right) \leq \operatorname{reg}\left(f_{1}, \ldots, f_{r-1}\right)+\operatorname{reg}(J) .
$$

Since $\operatorname{reg}(I)=\operatorname{reg}\left(f_{1}, \ldots, f_{r-1}\right)+\operatorname{deg} f_{r}-1$, this implies

$$
\operatorname{reg}\left(\left(\sum_{i=1}^{r-1} f_{i}\left(J: Q_{i}\right)\right) \cap f_{r}\left(J: Q_{r}\right)\right) \leq \operatorname{reg}(I)+\operatorname{reg}(J)+1 .
$$

Now, we apply Lemma 2.3 to the decomposition

$$
f_{1}\left(J: Q_{1}\right)+\cdots+f_{r}\left(J: Q_{r}\right)=\left(\sum_{i=1}^{r-1} f_{i}\left(J: Q_{i}\right)\right)+f_{r}\left(J: Q_{r}\right)
$$

and obtain

$$
\begin{aligned}
& \operatorname{reg}\left(f_{1}\left(J: Q_{1}\right)+\cdots+f_{r}\left(J: Q_{r}\right)\right) \\
& \leq \max \left\{\operatorname{reg}\left(\sum_{i=1}^{r-1} f_{i}\left(J: Q_{i}\right)\right),\right. \\
& \left.\quad \operatorname{reg}\left(f_{r}\left(J: Q_{r}\right)\right), \operatorname{reg}\left(\left(\sum_{i=1}^{r-1} f_{i}\left(J: Q_{i}\right)\right) \cap f_{r}\left(J: Q_{r}\right)\right)-1\right\} \\
& \quad \leq \operatorname{reg}(I)+\operatorname{reg}(J)
\end{aligned}
$$

by using (1), (2), (3).

Remark 3.2. The above proof would work in the case of more than two monomial complete intersections if we could prove a similar result to that in Corollary 2.2 . For instance, if we can prove

$$
\operatorname{reg}(I J: Q) \leq \operatorname{reg}(I)+\operatorname{reg}(J)
$$

for two monomial complete intersections $I, J$ and an arbitrary monomial ideal $Q$, then we can give a positive answer to the question of Conca and Herzog in the monomial case for $d=3$. We are unable to verify the above formula, although computations in concrete cases suggest its validity.

Now we will extend the second formula of Theorem 1.1 for any set of monomial complete intersections.

Theorem 3.3. Let $I_{1}, \ldots, I_{d}$ be arbitrary monomial complete intersections. Then

$$
\operatorname{reg}\left(I_{1} \cap \cdots \cap I_{d}\right) \leq \operatorname{reg}\left(I_{1}\right)+\cdots+\operatorname{reg}\left(I_{d}\right) .
$$

Proof. We will use induction on the number $n$ of variables and the sum

$$
s:=\operatorname{reg}\left(I_{1}\right)+\cdots+\operatorname{reg}\left(I_{d}\right) .
$$

First, we note that the cases $n=1$ and $s=1$ are trivial. 
Assume that $n \geq 2$ and $s \geq 2$. Let $x$ be an arbitrary variable of the polynomial ring $S$. It is easy to see that $\left(I_{1}, x\right), \ldots,\left(I_{d}, x\right)$ are monomial complete intersections and

$$
\left(I_{1} \cap \cdots \cap I_{d}, x\right)=\left(I_{1}, x\right) \cap \cdots \cap\left(I_{d}, x\right) .
$$

Therefore, using induction on $n$ we may assume that

$$
\operatorname{reg}\left(I_{1} \cap \cdots \cap I_{d}, x\right) \leq \operatorname{reg}\left(I_{1}, x\right)+\cdots+\operatorname{reg}\left(I_{d}, x\right) .
$$

If $x$ is a non-zerodivisor on $I_{1} \cap \cdots \cap I_{d}$ and if we assume that the intersection is irredundant, then $I_{j}: x=I_{j}$ and hence $\operatorname{reg}\left(I_{j}, x\right)=\operatorname{reg}\left(I_{j}\right)$ for all $j=1, \ldots, d$. In this case,

$$
\operatorname{reg}\left(I_{1} \cap \cdots \cap I_{d}\right)=\operatorname{reg}\left(I_{1} \cap \cdots \cap I_{d}, x\right) \leq \operatorname{reg}\left(I_{1}\right)+\cdots+\operatorname{reg}\left(I_{d}\right) .
$$

If $x$ is a zerodivisor on $I_{1} \cap \cdots \cap I_{d}$, we involve the ideal

$$
\left(I_{1} \cap \cdots \cap I_{d}\right): x=\left(I_{1}: x\right) \cap \cdots \cap\left(I_{d}: x\right) .
$$

If $I_{j}: x \neq I_{j}$, either $I_{j}: x=S\left(x \in I_{j}: x\right)$ or $I_{j}: x$ is a monomial complete intersection generated by the monomials obtained from the generators of $I_{j}$ by replacing the monomial divisible by $x$ by its quotient by $x$. In the latter case, we have $\operatorname{reg}\left(I_{j}: x\right)=\operatorname{reg}\left(I_{j}\right)-1$. Since there exists at least one ideal $I_{j}$ with $I_{j}: x \neq I_{j}$, the ideal $\left(I_{1} \cap \cdots \cap I_{d}\right): x$ is an intersection of monomial complete intersections such that the sum of their regularities is less than $s$. Using induction on $s$ we may assume that

$$
\begin{aligned}
\operatorname{reg}\left(\left(I_{1} \cap \cdots \cap I_{d}\right): x\right) & \leq \operatorname{reg}\left(I_{1}: x\right)+\cdots+\operatorname{reg}\left(I_{d}: x\right) \\
& \leq \operatorname{reg}\left(I_{1}\right)+\cdots+\operatorname{reg}\left(I_{d}\right)-1 .
\end{aligned}
$$

Now, from the exact sequence

$$
0 \longrightarrow S /\left(I_{1} \cap \cdots \cap I_{d}\right): x \stackrel{x}{\longrightarrow} S / I_{1} \cap \cdots \cap I_{d} \longrightarrow S /\left(I_{1} \cap \cdots \cap I_{d}, x\right) \longrightarrow 0
$$

we can deduce that

$$
\begin{aligned}
\operatorname{reg}\left(I_{1} \cap \cdots \cap I_{d}\right) & \leq \max \left\{\operatorname{reg}\left(\left(I_{1} \cap \cdots \cap I_{d}\right): x\right)+1, \operatorname{reg}\left(I_{1} \cap \cdots \cap I_{d}, x\right)\right\} \\
& \leq \operatorname{reg}\left(I_{1}\right)+\cdots+\operatorname{reg}\left(I_{d}\right) .
\end{aligned}
$$

\section{Counter-examples}

From now on we set $S:=k[x, y, z, t]$ for the homogeneous coordinate ring of $\mathbb{P}^{3}$. First, we will use the theory of liaison to give curves in $\mathbb{P}^{3}$ with sections in negative degrees. Recall that two curves $X$ and $Y$ in $\mathbb{P}^{3}$ are said to be directly linked by a complete intersection $Z$ if $I_{X}=I_{Z}: I_{Y}$, or equivalently $I_{Y}=I_{Z}: I_{X}$.

For any curve $Y$ we define

$$
\varepsilon(Y):=\min \left\{\mu \mid H^{0}\left(Y, \mathcal{O}_{Y}(\mu)\right) \neq 0\right\} .
$$

Note that if we denote the initial degree of a module $M$ by indeg $(M)$ with the convention $\operatorname{indeg}(0)=+\infty$, then

$$
\varepsilon(Y)=\min \left\{0, \operatorname{indeg}\left(H_{\mathfrak{m}}^{1}\left(S / I_{Y}\right)\right)\right\} .
$$

Lemma 4.1. Let $X$ and $Y$ be two curves in $\mathbb{P}^{3}$, directly linked by a complete intersection $Z$. Then, $\operatorname{reg}\left(S / I_{X}\right) \geq \operatorname{reg}\left(S / I_{Z}\right)$ if and only if $\varepsilon(Y)<0$. In this case,

$$
\operatorname{reg}\left(S / I_{X}\right)=\operatorname{reg}\left(S / I_{Z}\right)-\varepsilon(Y)-1 .
$$


Proof. Put $r:=\operatorname{reg}\left(S / I_{Z}\right)$. Then $a_{2}\left(S / I_{X}\right) \leq a_{2}\left(S / I_{Z}\right)=r-2$ because $X$ is strictly contained in $Z$. Hence $\operatorname{reg}\left(S / I_{X}\right) \geq r$ if and only if $a_{1}\left(S / I_{X}\right)+1 \geq r$. In this case, $\operatorname{reg}\left(S / I_{X}\right)=a_{1}\left(S / I_{X}\right)+1$, By liaison,

$$
H_{\mathfrak{m}}^{1}\left(S / I_{X}\right)_{\mu} \simeq H_{\mathfrak{m}}^{1}\left(S / I_{Y}\right)_{r-\mu-2} .
$$

Therefore, $a_{1}\left(S / I_{X}\right)=r-\operatorname{indeg}\left(H_{\mathfrak{m}}^{1}\left(S / I_{Y}\right)\right)-2$. Thus, $\operatorname{reg}\left(S / I_{X}\right) \geq r$ if and only if indeg $\left(H_{\mathfrak{m}}^{1}\left(S / I_{Y}\right)\right)<0$. In this case, $\operatorname{reg}\left(S / I_{X}\right)=r-\operatorname{indeg}\left(H_{\mathfrak{m}}^{1}\left(S / I_{Y}\right)\right)-1$.

For instance, we may take for $X$ a reduced irreducible curve whose regularity is at least equal to the sum of the two smallest degrees $d_{1}, d_{2}$ of the minimal generators of its defining ideal. By Lemma 4.1, the residual of $X$ in the complete intersection of degrees $d_{1}, d_{2}$ is a nonreduced curve $Y$ with

$$
\varepsilon(Y)=d_{1}+d_{2}-\operatorname{reg}\left(I_{X}\right)-2<0 .
$$

We will use curves with sections in negative degrees to construct complete intersection ideals $I, J$ for which $\operatorname{reg}(I \cap J)$ or $\operatorname{reg}(I J)$ is greater than $\operatorname{reg}(I)+\operatorname{reg}(J)$. The construction is based on the following theorems.

Theorem 4.2. Let $Y$ be a curve in $\mathbb{P}^{3}$ with $\varepsilon(Y)<0$ which is defined by at most 3 equations at the generic points of its irreducible components. Consider 3 elements $f_{1}, f_{2}, f_{3}$ in $I_{Y}$ such that $I_{Y}$ is the unmixed part of $\left(f_{1}, f_{2}, f_{3}\right)$ and $f_{1}, f_{2}$ is a regular sequence. Put $I=\left(f_{1}, f_{2}\right)$ and $J=\left(f_{3}\right)$. Then

$$
\operatorname{reg}(I \cap J)=\operatorname{reg}(I)+\operatorname{reg}(J)-\varepsilon(Y)-1 .
$$

Proof. Let $K=\left(f_{1}, f_{2}, f_{3}\right)$ and $\sigma=\operatorname{deg} f_{1}+\operatorname{deg} f_{2}+\operatorname{deg} f_{3}$. By [Ch, 0.6] we have

$$
\begin{aligned}
& a_{0}(S / K)=\sigma-\varepsilon(Y)-4, \\
& a_{1}(S / K)=\sigma-\operatorname{indeg}\left(I_{Y} / K\right)-4, \\
& a_{2}(S / K) \leq \sigma-\operatorname{indeg}(K)-5 .
\end{aligned}
$$

Since $\varepsilon(Y)<0, a_{0}(S / K) \geq a_{i}(S / K)+i$ for $i=1,2$. Therefore,

$$
\operatorname{reg}(S / K)=a_{0}(S / K)=\sigma-\varepsilon(Y)-4 .
$$

Applying Lemma 2.3 we get

$$
\operatorname{reg}(I \cap J)=\operatorname{reg}(K)+1=\sigma-\varepsilon(Y)-2=\operatorname{reg}(I)+\operatorname{reg}(J)-\varepsilon(Y)-1 .
$$

Theorem 4.3. Let $Y$ be a curve in $\mathbb{P}^{3}$ with $\varepsilon(Y)<0$ which is defined by at most 4 equations at the generic points of its irreducible components. Consider 4 elements $f_{1}, f_{2}, f_{3}, f_{4}$ in $I_{Y}$ such that $I:=\left(f_{1}, f_{2}\right)$ and $J:=\left(f_{3}, f_{4}\right)$ are complete intersection ideals and $I_{Y}$ is the unmixed part of $I+J$. Then

$$
\operatorname{reg}(I J)=\operatorname{reg}(I)+\operatorname{reg}(J)-\varepsilon(Y)-1 .
$$

Proof. Consider the exact sequence

$$
0 \rightarrow(I \cap J) / I J \rightarrow S / I J \rightarrow S /(I \cap J) \rightarrow 0 .
$$

As $\operatorname{Tor}_{1}^{S}(S / I, S / J) \simeq(I \cap J) / I J$ and $\operatorname{depth}(S /(I \cap J))>0$ one has

$$
H_{\mathfrak{m}}^{0}(S / I J) \simeq H_{\mathfrak{m}}^{0}\left(\operatorname{Tor}_{1}^{S}(S / I, S / J)\right) .
$$


Put $\sigma=\operatorname{deg} f_{1}+\operatorname{deg} f_{2}+\operatorname{deg} f_{3}+\operatorname{deg} f_{4}$. By [Ch, 5.9], $H_{\mathfrak{m}}^{0}\left(\operatorname{Tor}_{1}^{S}(S / I, S / J)\right)$ is the graded $k$-dual of $H_{\mathfrak{m}}^{1}\left(S / I_{Y}\right)$ up to a shift in degrees by $\sigma-4$. Therefore, $H_{\mathfrak{m}}^{0}(S / I J)_{\mu} \simeq H_{\mathfrak{m}}^{1}\left(S / I_{Y}\right)_{\sigma-4-\mu}$. This implies

$$
a_{0}(S / I J)=\sigma-\operatorname{indeg}\left(H_{\mathfrak{m}}^{1}\left(S / I_{Y}\right)\right)-4=\sigma-\varepsilon(Y)-4 .
$$

Modifying the generators of $I$ and $J$, we may assume that $f_{1} f_{3}, f_{2} f_{4}$ is a regular sequence, which shows that

$$
a_{2}(S / I J) \leq a_{2}\left(S /\left(f_{1} f_{3}, f_{2} f_{4}\right)\right)-1=\sigma-5 .
$$

By [Ch, 3.1 (iii)] one has $H_{\mathfrak{m}}^{1}((I \cap J) / I J)_{\mu}=0$ for $\mu>\sigma-4$. Therefore, if $\mu>\sigma-4$, one has an exact sequence

$$
0 \longrightarrow H_{\mathfrak{m}}^{1}(S / I J)_{\mu} \longrightarrow H_{\mathfrak{m}}^{1}(S / I \cap J)_{\mu} \longrightarrow H_{\mathfrak{m}}^{2}((I \cap J) / I J)_{\mu} \longrightarrow 0 .
$$

By [Ch, 3.1 (ii)], $H_{\mathfrak{m}}^{2}((I \cap J) / I J)_{\mu} \simeq H_{\mathfrak{m}}^{0}(S / I+J)_{\mu}$ for $\mu>\sigma-4$. Since $S / I$ and $S / J$ are Cohen-Macaulay rings, using the exact sequence

$$
0 \longrightarrow S / I \cap J \longrightarrow S / I \oplus S / J \longrightarrow S / I+J \longrightarrow 0
$$

we also have $H_{\mathfrak{m}}^{1}(S / I \cap J)_{\mu} \simeq H_{\mathfrak{m}}^{0}(S / I+J)_{\mu}$. Therefore, $H_{\mathfrak{m}}^{1}(S / I J)_{\mu}=0$ for $\mu>\sigma-4$. Hence

$$
a_{1}(S / I J) \leq \sigma-4 .
$$

As $\varepsilon(Y)<0, a_{i}(S / I J)+i \leq a_{0}(S / I J)$ for $i=1,2$. So we get

$$
\operatorname{reg}(I J)=a_{0}(S / I J)+1=\sigma-\varepsilon(Y)-4=\operatorname{reg}(I)+\operatorname{reg}(J)-\varepsilon(Y)-1 .
$$

We will now study a specific class of curves with sections in negative degrees.

Consider first a monomial curve $C$ parameterized by $\left(1: \theta: \theta^{m n}: \theta^{m(n+1)}\right)$ for $m, n \geq 2$. Note that $\operatorname{reg}\left(I_{C}\right)=m n$ by $\overline{\mathrm{BCFH}}$, Corollary 5.1]. Let $X$ be the union of $C$ and the line $\{x=z=0\}$. On one hand, $\operatorname{reg}\left(I_{X}\right) \geq m n+1$ because $x y^{m n}-x^{m n} z$ is a minimal generator of $I_{X}=I_{C} \cap(x, z)$. On the other hand, it is easy to check that

$$
I_{C}+(x, z)=\left(x, z, y^{m} t^{n-1}, y^{2 m} t^{n-2}, \ldots, y^{n m}\right)
$$

and hence $\operatorname{reg}\left(I_{C}+(x, z)\right)=m n$. By Lemma 2.3, this implies reg $\left(I_{X}\right) \leq \operatorname{reg}\left(I_{C}\right)+$ $1=m n+1$ (one can also use [Si, 1.8] to show $\operatorname{reg}\left(I_{X}\right) \leq m n+1$ ). Therefore, $\operatorname{reg}\left(I_{X}\right)=m n+1$.

Let $Y$ be the direct link of $X$ by the complete intersection defined by the ideal $\left(x^{m} t-y^{m} z, z^{n+1}-x t^{n}\right)$. By Lemma 4.1 .

$$
\varepsilon(Y)=(m+1)+(n+1)-\operatorname{reg}\left(I_{X}\right)-2=-(m-1)(n-1) .
$$

One has $I_{Y}=\left(x^{m} t-y^{m} z\right)+(z, t)^{n}$. To prove this note that $\left(x^{m} t-y^{m} z\right)+(z, t)^{n}$ defines a locally complete intersection scheme supported on the line $z=t=0$, has positive depth and degree (or multiplicity) at least $n$. Then the containment

$$
I_{Z}=I_{X} \cap I_{Y} \subseteq I_{X} \cap\left(\left(x^{m} t-y^{m} z\right)+(z, t)^{n}\right)
$$

and the fact that

$$
\operatorname{deg}\left(I_{Z}\right)=(m+1)(n+1)=\operatorname{deg}\left(I_{X}\right)+n
$$

forces $I_{Y}$ to coincide with the unmixed ideal $\left(x^{m} t-y^{m} z\right)+(z, t)^{n}$. It is also easy to provide a minimal free $S$-resolution of the ideal $\left(x^{m} t-y^{m} z\right)+(z, t)^{n}$, and show these facts along the same line as in the proof of [CD, 2.4]. 
The above curve $Y$ can be used to construct counter-examples to the inequalities raised in the introduction.

Example 4.4. Let $I=\left(t^{n}, z^{n}\right)$ and $J=\left(x^{m} t-y^{m} z\right)$. It is easy to check that $I_{Y}=\left(x^{m} t-y^{m} z\right)+(z, t)^{n}$ is the saturation of $\left(t^{n}, z^{n}, x^{m} t-y^{m} z\right)$. Therefore, we may apply Theorem 4.2 and obtain

$$
\operatorname{reg}(I \cap J)=\operatorname{reg}(I)+\operatorname{reg}(J)+(m-1)(n-1)-1 .
$$

Thus, $\operatorname{reg}(I \cap J)>\operatorname{reg}(I)+\operatorname{reg}(J)$ if and only if $m n>m+n$ or equivalently $(m, n) \neq(2,2)$.

Example 4.5. Let $I=\left(t^{n}, z^{n}\right)$ and $J=\left(x^{m} t-y^{m} z, t^{n}\right)$. Then $I_{Y}$ is the saturation of $I+J$. By Theorem 4.3 we have

$$
\operatorname{reg}(I J)=\operatorname{reg}(I)+\operatorname{reg}(J)+(m-1)(n-1)-1 .
$$

Therefore, $\operatorname{reg}(I J)>\operatorname{reg}(I)+\operatorname{reg}(J)$ if and only if $(m, n) \neq(2,2)$.

\section{ACKNOWLEDGMENT}

The first author is grateful to the Institute of Mathematics in Hanoi for its hospitality while this work was completed.

\section{REFERENCES}

[BaM] D. Bayer and D. Mumford, What can be computed in algebraic geometry? In: D. Eisenbud and L. Robbiano (eds.), Computational Algebraic Geometry and Commutative Algebra, Proceedings, Cortona 1991, Cambridge University Press, 1993, 1-48. MR.1253986 (95d:13032)

$[\mathrm{BCFH}]$ H. Bresinsky, F. Curtis, M. Fiorentini, L. T. Hoa, On the structure of local cohomology modules for projective monomial curves in $\mathbb{P}^{3}$. Nagoya Math. J. 136 (1994), 81-114. MR1309382 (96b:14040)

[BrH] W. Bruns and J. Herzog, On multigraded resolutions, Math. Proc. Camb. Phil. Soc. 118 (1995), 245-257. MR 1341789 (96g:13013)

[Ch] M. Chardin, Regularity of ideals and their powers, Prépublication 364, Institut de Mathematiques de Jussieu, 2004.

[CD] M. Chardin, C. D'Cruz, Castelnuovo-Mumford regularity: examples of curves and surfaces, J. Algebra 270 (2003), 347-360. MR2016666 (2004m:13036)

[CU] M. Chardin, B. Ulrich, Liaison and Castelnuovo-Mumford regularity, Amer. J. Math. 124 (2002), 1103-1124. MR1939782 (2004c:14095)

[CoH] A. Conca and J. Herzog, Castelnuovo-Mumford regularity of products of ideals, Collect. Math. 54 (2003), 137-152. MR.1995137(2004k:13020)

[DS] H. Derksen and J. Sidman, A sharp bound for the Castelnuovo-Mumford regularity of subspace arrangements, Adv. Math. 172 (2002), no. 2, 151-157. MR.1942401 (2003m:13013)

[E] D. Eisenbud, Commutative algebra with a view toward algebraic geometry, Springer, 1994. MR1322960 (97a:13001)

[HT] L. T. Hoa and N. V. Trung, On the Castelnuovo-Mumford regularity and the arithmetic degree of monomial ideals, Math. Zeits. 229 (1998), 519-537. MR.1658557 (99k:13034)

[M] N. C. Minh, On Castelnuovo-Mumford regularity of products of monomial ideals, Acta Math. Vietnam 30 (2005), 203-209. MR2192521(2006i:13034)

[Si] J. Sidman, On the Castelnuovo-Mumford regularity of products of ideal sheaves, Adv. Geom. 2 (2002), 219-229. MR 1924756 (2003f:13021)

[St] B. Sturmfels, Four counter examples in combinatorial algebraic geometry, J. Algebra 230 (2000), 282-294. MR:1774768 (2001g:13047) 
Institut de Mathématiques de Jussieu, CNRS \& Université Paris Vi, Paris, France

E-mail address: chardin@math.jussieu.fr

Department of Mathematics, University of Education, 136 XuÂn Thuy, Hanoi, VietNAM

E-mail address: ngcminh@gmail.com

Institute of Mathematics, Viên ToÁn Hoc, 18 Hò̀ng Quôc Viêt, 1037 Hanoi, Vietnam

E-mail address: nvtrung@math.ac.vn 